\title{
Empilements de cercles et discrétisation quasiconforme: comportement asymptotique des rayons
}

\author{
F. Mathéus \\ Université de Bretagne-Sud, \\ Laboratoire de Mathématiques et d'Applications des Mathématiques, \\ 1 rue de la Loi, 56000, Vannes, France \\ Frederic.Matheus@univ-ubs.fr
}

\begin{abstract}
In a discretization scheme for conformal mappings with circle packings previously considered by Colin de Verdière and the author, a two-term asymptotic expansion of the radii of the image circles is obtained, with a control on the error. The vanishing of the second term characterizes Doyle spirals.
\end{abstract}

Résumé. Dans le cadre d'une méthode d'approximation de fonctions holomorphes au moyen d'empilements de cercles étudiée antérieurement par Colin de Verdière et l'auteur, nous obtenons un développement asymptotique à deux termes des rayons des cercles de l'empilement image, avec majoration du reste. L'annulation du second terme donne une caractérisation des empilements de type exponentiel.

\section{Introduction}

Depuis une dizaine d'années, l'utilisation des empilements de cercles pour discrétiser des fonctions holomorphes a fait l'objet d'abondants travaux. C'est d'abord l'uniformisation de Riemann d'un ouvert simplement connexe borné de $\mathbf{C}$ qui a été étudiée par Rodin et Sullivan. Suivant une idée de Thurston (voir [Th]), ils en ont construit des approximations quasiconformes au moyen d'empilements de cercles finis à combinatoire hexagonale (voir $[R S])$. Par la suite, Stephenson, He, Rodin et Schramm ont généralisé le théorème d'approximation à des empilements de cercles dont la combinatoire et les rayons sont variables (voir [S1], [S2], [HR], et [HS]).

L'extension de ce procédé de discrétisation aux fonctions holomorphes dont la dérivée ne s'annule pas, au moyen d'empilements immergés, a été menée en parallèle par Carter 
et Rodin d'une part (voir [CR]), et par Colin de Verdière et l'auteur d'autre part (voir $[\mathrm{CM}])$. Enfin, le cas où la dérivée s'annule a été étudié par Dubejko qui utilise pour cela des empilements ramifiés (voir [D1]).

Dans toutes les situations envisagées, il est rapidement apparu que les rayons des cercles des empilements construits permettaient d'accéder directement au module de la dérivée de la fonction discrétisée. L'idée_-assez intuitive-est la suivante: si $z \in \mathbf{C}$ est un point en lequel la dérivée de $f$ ne s'annule pas, et si $C$ est un cercle d'un empilement source de rayon $\varepsilon$ et contenant $z$, alors le rayon $r$ du cercle $C^{\prime}$ de l'empilement image correspondant à $C$ est équivalent à $\varepsilon\left|f^{\prime}(z)\right|$. Dans la situation de [CM] est même obtenue l'estimation $r=\varepsilon\left|f^{\prime}(z)\right|+O\left(\varepsilon^{2}\right)$.

Le but de ce travail est de comprendre quelles informations supplémentaires sur $f$ on peut récupérer à partir des rayons des cercles des empilements images. On montre comment obtenir, dans le cadre du schéma d'approximation étudié dans [CM], un développement asymptotique de la forme:

$$
r=\varepsilon\left|f^{\prime}(z)\right|+\varepsilon^{3} h(z)+O\left(\varepsilon^{4} \log \varepsilon\right)
$$

(cf. théorème 2.1). Le coefficient de $\varepsilon^{3}$ possède une interprétation géométrique. Son annulation nous permet de caractériser les fonctions entières $f$ pour lesquelles on a une formule exacte $r=\varepsilon\left|f^{\prime}(z)\right|$ (cf. corollaire 2.2): $f$ est alors ou bien de la forme $f(z)=a z+b$, ou bien de la forme $f(z)=e^{a z+b}+c$. L'absence de coefficient en $\varepsilon^{2}$ permet d'avancer un début d'explication à un phénomène de rapidité de convergence observé par Dubejko et Stephenson (voir la section 4, exp. \#3, p. 329, de [DS]).

Décrivons brièvement la structure de ce texte. Dans la section suivante on rappelle le schéma d'approximation étudié dans $[\mathrm{CM}]$ afin de donner un énoncé précis du théorème principal. Son corollaire y est énoncé et démontré. La section 3 est consacrée à une méthode de déformation d'empilements (de cercles) dont la courbure varie. Il s'agit d'une variante de celle utilisée dans [CM]. Des estimations a priori sur les rayons des cercles, de type principe du maximum, sont obtenues par voie probabiliste dans la section 4, au moyen de marches aléatoires et de martingales. Elles permettent, dans la section 5 , de mener à bien la preuve du théorème principal. Celle-ci repose sur la discrétisation d'un problème de Dirichlet étudiée dans le cadre de la méthode des volumes finis.

\section{2. Énoncé du théorème principal}

Commençons par rappeler la définition d'un empilement de cercles. Soit $\mathcal{T}$ une triangulation (finie ou infinie) d'un disque topologique et $S$ l' ensemble des sommets de $\mathcal{T}$. Soit $r=\left(r_{s}\right)_{s \in S} \in\left(\mathbf{R}_{+}^{*}\right)^{S}$. On munit chaque triangle de $\mathcal{T}$ de la métrique euclidienne $g_{r}$ qui au côté $s s^{\prime}$ affecte la longueur $r_{s}+r_{s^{\prime}}$. On obtient ainsi une variété riemannienne plate à singularités coniques, notée $\mathcal{T}_{r}$ (voir [Tr]). Soit $s$ un sommet d'un triangle $T$ de $\mathcal{T}$. Si $\alpha(s, T)$ désigne l'angle en $s$ dans le triangle $T$ et si $s$ est un sommet intérieur, alors la courbure en $s$ est:

$$
K_{s}(r)=2 \pi-\sum_{s \in T} \alpha(s, T)
$$




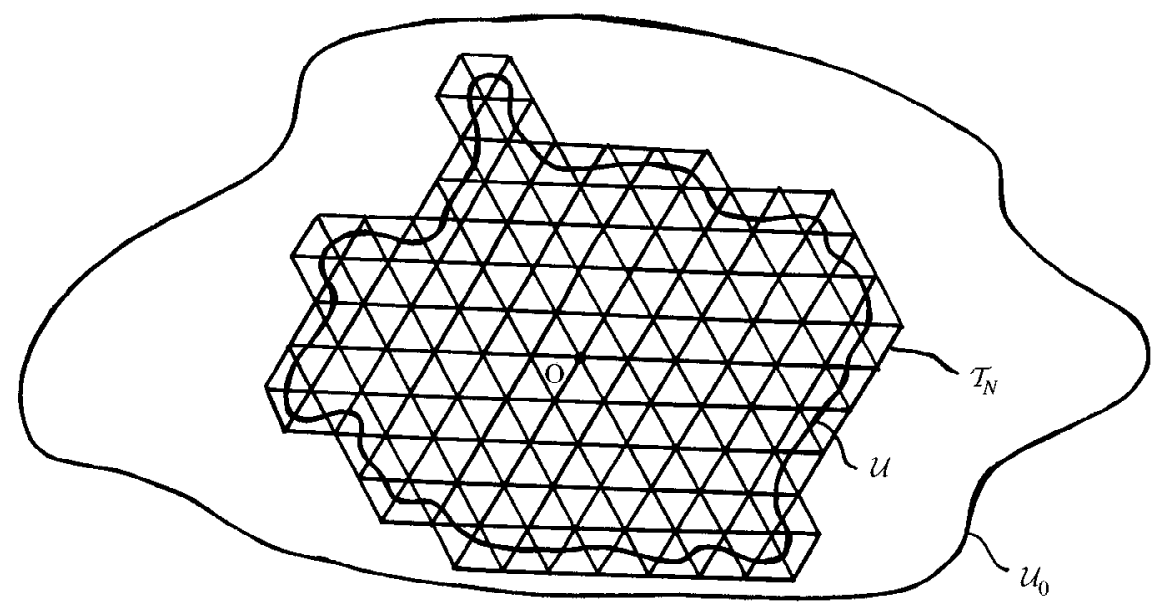

Fig. 1. Les ouverts $\mathcal{U}$ et $\mathcal{U}_{0}$, et la triangulation $\mathcal{T}_{N}$.

La famille de cercles $\mathcal{C}=\left\{C_{s}, s \in S\right\}$, où $C_{s}$ est le cercle de $\mathcal{T}_{r}$ de centre $s$, de rayon $r_{s}$, possède la propriété suivante: les cercles $C_{s}$ et $C_{s^{\prime}}$ sont tangents si et seulement si l'arête $s s^{\prime}$ appartient au 1-squelette $\mathcal{T}^{1}$ de la triangulation $\mathcal{T}$.

$\mathrm{Si}$, pour tout sommet intérieur $s$, on a $K_{s}(r)=0$, alors la variété $\mathcal{T}_{r}$ est immergée isométriquement dans $\mathbf{R}^{2}$ (mais non plongée a priori). On dit alors que $\mathcal{C}$ est un empilement de cercles immergé de combinatoire $\mathcal{T}^{1}$.

Soit $\mathcal{U}$ un ouvert borné, connexe, simplement connexe, contenant 0 , à bord de classe $C^{3}$, et soit $f$ une fonction holomorphe définie sur un voisinage $\mathcal{U}_{0}$ de l'adhérence $\overline{\mathcal{U}}$ de $\mathcal{U}$, et dont la dérivée ne s'annule pas. Pour $N \geq 1$, soit $\mathcal{T}_{N}(\mathbf{C})$ la triangulation de $\mathbf{C}$ par des triangles équilatéraux de côté $1 / N$, dont l'ensemble des sommets contient 0 et $1 / N$. Soit $\mathcal{T}_{N}$ la sous-triangulation de $\mathcal{T}_{N}(\mathbf{C})$ constituée des triangles de $\mathcal{T}_{N}(\mathbf{C})$ qui rencontrent $\overline{\mathcal{U}}$. Si $N$ est assez grand $\left(\geq N_{0}\right), \mathcal{T}_{N}$ est une triangulation d'un disque topologique (cf. fig. 1). On note $S_{N}$ l'ensemble des sommets de $\mathcal{T}_{N}$, et $H_{N}$ l'empilement constitué des cercles de rayon $1 / 2 N$ et dont les centres sont les sommets de $\mathcal{T}_{N}$. On constate que la combinatoire de $H_{N}$ n'est autre que $\mathcal{T}^{1}$.

On construit un nouvel empilement, noté $\tilde{H}_{N}$, de la façon suivante. Il doit vérifier les deux conditions (i) et (ii) ci-dessous:

(i) la combinatoire de $\tilde{H}_{N}$ est $\mathcal{T}^{1}$;

(ii) les rayons des cercles du bord de $\tilde{H}_{N}$ sont donnés par $(1 / 2 N)\left|f^{\prime}(s)\right|$ où $s$ décrit l'ensemble des sommets frontière de $\mathcal{T}_{N}$.

D'après le premier théorème de la section 4 de [C], il existe un empilement de cercles vérifiant les conditions (i) et (ii) ci-dessus, et cet empilement est unique à isométrie près. Notons $C_{N}^{s}$ le cercle de $H_{N}$ centré en $s \in S_{N}$ et $r_{N}^{s}$ le rayon du cercle $\tilde{C}_{N}^{s}$ de $\tilde{H}_{N}$ correspondant à $C_{N}^{s}$. L'empilement $\tilde{H}_{N}$ sera parfaitement déterminé si l'on exige qu'il 
satisfasse la condition suivante, dite de normalisation:

(iii) le cercle $\tilde{C}_{N_{-}}^{0}$ est centré en $f(0)$, et le cercle $\tilde{C}_{N}^{1 / N}$ est centré sur la demi-droite $f(0)+\mathbf{R}_{+} f^{\prime}(0)$.

Dans toute la suite, $\tilde{H}_{N}$ désignera l'unique empilement de cercles du plan satisfaisant aux conditions (i), (ii) et (iii) ci-dessus (cf. [CM] pour des figures).

Considérons maintenant l'application $f_{N}$ définie sur $\mathcal{T}_{N}$, affine sur chaque triangle de $\mathcal{T}_{N}$, et envoyant le centre de tout cercle $C$ de $H_{N}$ sur le centre du cercle correspondant $\tilde{C}$ de $\tilde{H}_{N}$. Dans [CM], Colin de Verdière et l'auteur démontrent que la suite des fonctions $f_{N}$ converge uniformément sur $\overline{\mathcal{U}}$ vers $f$, et obtiennent l'estimation suivante, uniforme $\operatorname{sur} \overline{\mathcal{U}}$ :

$$
r_{N}^{s}=\frac{\left|f^{\prime}(s)\right|}{2 N}+O\left(\frac{1}{N^{2}}\right) .
$$

Soit $\check{f}: \mathcal{U}_{0} \longrightarrow \mathbf{R}$ la fonction définie par

$$
\check{f}=\frac{1}{36}\left|\frac{f^{\prime \prime}}{f^{\prime}}\right|^{4}\left[\Re\left(\frac{f^{\prime} f^{\prime \prime \prime}}{f^{\prime \prime 2}}\right)-\left|\frac{f^{\prime} f^{\prime \prime \prime}}{f^{\prime \prime 2}}\right|^{2}\right] .
$$

Il est aisé de vérifier que $\check{f}$ est une fonction $C^{\infty}$ sur $\mathcal{U}_{0}$. Notons $\Delta$ l'opérateur $-\partial^{2} / \partial x^{2}-\partial^{2} / \partial y^{2}$, et soit $g: \overline{\mathcal{U}} \longrightarrow \mathbf{R}$ la fonction de classe $C^{2}$ définie par:

$$
\begin{cases}\Delta g=\check{f} & \operatorname{sur} \mathcal{U} \\ g=0 & \operatorname{sur} \partial \mathcal{U}\end{cases}
$$

(voir le théorème 6.14 p. 107 de [GT] pour la régularité de $g$ ).

Le théorème suivant, qui constitue le résultat principal de ce texte, précise le comportement asymptotique des rayons des cercles de l'empilement $\tilde{H}_{N}$ :

Théorème 2.1. Pour tout $s \in S_{N}$, on $a$

$$
r_{N}^{s}=\frac{\left|f^{\prime}(s)\right|}{2 N}\left(1+\frac{g(s)}{N^{2}}\right)+O\left(\frac{\log N}{N^{4}}\right) \quad \text { quand } \quad N \longrightarrow \infty
$$

uniformément sur $S_{N}$.

Le corollaire suivant caractérise le cas où l'on a une formule exacte:

Corollaire 2.2. Soit $\mathcal{U}$ un ouvert de $\mathbf{C}$ comme ci-avant et $f: \mathbf{C} \longrightarrow \mathbf{C}$ une fonction entière dont la dérivée ne s'annule pas. Les propriétés suivantes sont équivalentes:

(i) $\forall N \geq N_{0}, \forall s \in S_{N}, r_{N}^{s}=\left|f^{\prime}(s)\right| / 2 N$;

(ii) $\forall N \geq N_{0}, \forall s \in S_{N}, r_{N}^{s}=\left|f^{\prime}(s)\right| / 2 N+O\left((\log N) / N^{4}\right)$;

(iii) $\exists(a, b, c) \in \mathbf{C}^{*} \times \mathbf{C}^{2}$ tel que ou bien $f(z)=a z+b$, ou bien $f(z)=e^{a z+b}+c$.

Démonstration du corollaire 2.2. L'implication (i) $\Rightarrow$ (ii) est banale. Prouvons (ii) $\Rightarrow$ (iii). Considérons l'ouvert $\Omega=\left\{z \in \mathcal{U} \mid f^{\prime \prime}(z) \neq 0\right\}$, et supposons-le non vide. Soit 
$\tilde{f}$ la fonction holomorphe sur $\Omega$ définie par $\tilde{f}=f^{\prime} f^{\prime \prime \prime} / f^{\prime \prime 2}$. L'hypothèse entraine que la fonction $g$ introduite ci-avant est nulle sur une partie dense de $\mathcal{U}$-à savoir la réunion des $S_{N}$ - donc sur $\mathcal{U}$, de sorte que $\Re \tilde{f}-|\tilde{f}|^{2}=0$ sur $\Omega$. Ainsi, la fonction $\tilde{f}$ prend ses valeurs dans un cercle, donc est constante d'après le théorème de l'image ouverte. Notons $\alpha$ la valeur de $\tilde{f}$. On observe que $\left(f^{\prime} / f^{\prime \prime}\right)^{\prime}=1-\tilde{f}=1-\alpha$, ce qui donne $f^{\prime}(z)=[(1-\alpha) z+\beta] f^{\prime \prime}(z)$ sur $\Omega$, donc sur $\mathbf{C}$ par prolongement analytique. Comme $f^{\prime}$ ne s'annule pas sur $\mathbf{C}$, on a $1-\alpha=0$ et $\beta \neq 0$, donc $f^{\prime \prime}=(1 / \beta) f^{\prime}$, puis $f^{\prime}(z)=\exp ((1 / \beta) z+\gamma)$, ce qui prouve le point (iii) lorsque $\Omega$ n'est pas vide. Si $\Omega$ est vide, alors $f^{\prime \prime}=0$ sur $\mathcal{U}$, donc sur $\mathbf{C}$, et $f(z)=a z+b$, ce qui achève la preuve de (iii).

Prouvons (iii) $\Rightarrow$ (i). Si $f(z)=a z+b$ alors tous les cercles de $\tilde{H}_{N}$ ont pour rayon $|a| / 2 N=\left|f^{\prime}(z)\right| / 2 N$. Si $f(z)=e^{a z+b}+c$, l'assertion (i) résulte de la section III de $[\mathrm{CM}]$.

\section{Déformation d'empilements non plats}

Rappelons que notre but est d'évaluer, de manière plus précise que dans [CM], l'écart entre la collection des rayons des cercles de l'empilement $\tilde{H}_{N}$ et la collection $\left\{\left|f^{\prime}(s)\right| / 2 N\right.$, $s$ sommet de $\mathcal{T}_{N}$.

L'observation de départ est la suivante: lorsqu'on remplace le rayon $r_{N}^{s}$ de chaque cercle de l'empilement $\tilde{H}_{N}$ par $\left|f^{\prime}(s)\right| / 2 N$, on crée, en chaque sommet $s$ de $\mathcal{T}_{N}$, de la courbure au sens du début de la section précédente, notée $\breve{K}_{N}^{s}$, et que l'on sait estimer grâce au lemme 1 de la section VI de $[\mathrm{CM}]: \check{K}_{N}^{s}=O\left(1 / N^{4}\right)$. En ce sens, il est apparu peu de courbure. L'idée est alors de faire varier continûment la courbure entre celle que l'on a créée, c'est-à-dire $\check{K}_{N}^{s}$, et celle correspondant à l'empilement $\tilde{H}_{N}$, à savoir 0 , et ce tout en contrôlant les rayons des cercles.

Notons $\tilde{\mathcal{T}}_{N}^{0}$ la variété riemannienne plate à singularités (éventuelles) coniques définie à partir de la triangulation $\mathcal{T}_{N}$ et de la collection $\left\{\left|f^{\prime}(s)\right| / 2 N\right\}_{s \in S_{N}}$, et $\tilde{\mathcal{T}}_{N}^{1}$ la variété riemannienne plate-sans singularité-associée à l'empilement $\tilde{H}_{N}$. On se propose de réaliser $\tilde{\mathcal{T}}_{N}^{1}$ comme le temps 1 d'une déformation de la variété $\tilde{\mathcal{T}}_{N}^{0}$. On note $B_{N}$ l'ensemble des sommets du bord de $\mathcal{T}_{N}$ et $I_{N}$ l'ensemble des sommets intérieurs. On a bien sûr $S_{N}=B_{N} \bigsqcup I_{N}$. Cette déformation est fournie par la

Proposition 3.1. Pour tout $t \in[0,1]$, il existe une unique collection de rayons $\left\{r_{N}^{s}(t)\right\}_{s \in S_{N}} \in\left(\mathbf{R}_{+}^{*}\right)^{S_{N}}$ vérifiant les propriétés suivantes:

(i) pour tout $s \in I_{N}$, la courbure en s à l'instant t est donnée par $K_{N}^{s}(t)=(1-t) \check{K}_{N}^{s}$;

(ii) pour tout $s \in B_{N}, r_{N}^{s}(t)=\left|f^{\prime}(s)\right| / 2 N$.

On note $\tilde{\mathcal{T}}_{N}^{t}$ la variété riemannienne plate à singularités coniques ainsi obtenue. Bien entendu, pour $t=0$ et 1 , cette notation est cohérente avec celle introduite précédemment.

Preuve de la proposition 3.1. Il s'agit d'une relecture des sections 2 et 4 de [C]. On fixe $N \geq N_{0}$ et $t \in[0,1]$. Posons $u_{N}^{s}=\log r_{N}^{s}$, et fixons $u_{N}^{s}=\log \left(\left|f^{\prime}(s)\right| / 2 N\right)$ pour $s \in B_{N}$. 
On construit, exactement comme dans la section 2 de [C], une fonctionnelle $F_{t}$ : $\mathbf{R}^{I_{N}} \longrightarrow \mathbf{R}$, positive, strictement convexe, propre, et dont la différentielle est donnée, pour $u_{N}=\left(u_{N}^{s}\right)_{s \in I_{N}} \in \mathbf{R}^{I_{N}}$, par:

$$
d F_{t}\left(u_{N}\right)=\sum_{s \in I_{N}}\left[K_{N}^{s}\left(u_{N}\right)-K_{N}^{s}(t)\right] d u_{N}^{s} .
$$

Pour pouvoir construire $F_{t}$, la seule condition à vérifier - outre la fermeture de la forme différentielle figurant au second membre de l'équation ci-dessus - est l'existence d'un système d'angles $\left\{\alpha^{t}(s, T) \in\right] 0, \pi\left[, s \in S_{N}, T\right.$ triangle de $\mathcal{T}_{N}$ contenant $\left.s\right\}$ tel que:

(a) $\forall s \in I_{N}, \sum_{T \ni s} \alpha^{t}(s, T)=2 \pi-K_{N}^{s}(t)$;

(b) $\forall T \in \mathcal{T}_{N}, \sum_{s \in T} \alpha^{t}(s, T)=\pi$

(comparer avec la définition du Section 2 de [C]). La condition (a) exprime que la courbure au sommet $s \in I_{N}$ vaut précisément $K_{N}^{s}(t)$, et la condition (b), que chaque triangle pris individuellement est euclidien. Il suffit pour cela de prendre $\alpha^{t}(s, T)=$ $(1-t) \alpha^{0}(s, T)+t \alpha^{1}(s, T)$, où $\alpha^{0}(s, T)$ (resp. $\left.\alpha^{1}(s, T)\right)$ désigne l'angle en $s$ dans le triangle $T$ de la variété $\tilde{\mathcal{T}}_{N}^{0}$ (resp. $\tilde{\mathcal{T}}_{N}^{1}$ ).

Les propriétés de $F_{t}$ - stricte convexité, propreté, positivité - lui assurent l'existence d'un unique point critique $u_{N}(t) \in \mathbf{R}^{I_{N}}$, et la collection de rayons définie par $r_{N}^{s}(t)=$ $e^{u_{N}^{s}(t)}$ convient.

On va maintenent interpréter la famille $\left\{(d / d t) u_{N}^{s}(t)\right\}_{s \in S_{N}}$ comme solution d'un problème de Dirichlet discret. Pour ce faire, on dérive la relation du (i) de la proposition par rapport au paramètre $t$. Comme la courbure en $s$ ne dépend que des rayons en $s$ et aux sommets voisins, il vient:

$$
\forall t \in[0,1], \quad \forall s \in I_{N}, \quad \sum_{s^{\prime} \in S_{N}, d_{c}\left(s, s^{\prime}\right) \leq 1} \frac{\partial K_{N}^{s}\left[u_{N}(t)\right]}{\partial u_{N}^{s^{\prime}}} \dot{u}_{N}^{s^{\prime}}(t)=-\check{K}_{N}^{s},
$$

où $d_{c}$ désigne la distance combinatoire dans le graphe $\mathcal{T}_{N}$. Une formule explicite pour les dérivées partielles de la courbure $K_{N}^{s}$ est donnée à la fin de la section 5.1, dans la preuve du corollaire 5.1.2.

À toute fonction $\varphi \in \mathbf{R}^{I_{N}}$ prolongée par $0 \operatorname{sur} B_{N}$ on associe la fonction $\Delta_{N, t} \varphi=$ $\Delta_{N} \varphi \in \mathbf{R}^{I_{N}}$ définie par:

$$
\forall s \in I_{N}, \quad \Delta_{N} \varphi(s)=\sum_{s^{\prime} \in S_{N}, d_{c}\left(s, s^{\prime}\right) \leq 1} \frac{\partial K_{N}^{s}\left[u_{N}(t)\right]}{\partial u_{N}^{s^{\prime}}} \varphi\left(s^{\prime}\right) .
$$

Les relations suivantes (où l'on note $s^{\prime} \sim s$ pour $s^{\prime}$ voisin de $s$ ):

$$
\begin{aligned}
& \sum_{d_{c}\left(s, s^{\prime}\right) \leq 1} \frac{\partial K_{N}^{s}\left(u_{N}\right)}{\partial u_{N}^{s^{\prime}}}=\frac{\partial K_{N}^{s}}{\partial u_{N}^{s}}+\sum_{s^{\prime} \sim s} \frac{\partial K_{N}^{s}}{\partial u_{N}^{s^{\prime}}}=0 ; \quad \frac{\partial K_{N}^{s}}{\partial u_{N}^{s}}>0, \\
& \frac{\partial K_{N}^{s}}{\partial u_{N}^{s^{\prime}}}<0 \quad \text { si } \quad s^{\prime} \sim s, \quad \frac{\partial K_{N}^{s}}{\partial u_{N}^{s^{\prime}}}=\frac{\partial K_{N}^{s^{\prime}}}{\partial u_{N}^{s}} \quad \text { si } \quad s^{\prime} \sim s,
\end{aligned}
$$


font de l'opérateur $\Delta_{N}$ un laplacien discret sur $\mathbf{R}^{I_{N}}$, c'est-à-dire un endomorphisme symétrique défini positif de $\mathbf{R}^{I_{N}}$.

En résumé, le vecteur $\dot{u}_{N}(t)=\left\{\dot{u}_{N}^{s}(t)\right\}_{s \in S_{N}}$ est solution du problème de Dirichlet discret suivant:

$$
\begin{cases}\Delta_{N} \dot{u}_{N}(s)=-\check{K}_{N}^{s} & \text { pour tout } \\ \dot{u}_{N}^{s}=0 & \text { pour tout } \quad s \in I_{N} \\ & s \in B_{N}\end{cases}
$$

(comparer avec la section V de [CM], ainsi qu'avec la section III de [M]).

Le lemme suivant permet d'estimer le second membre de cette E.D.P. discrète:

Lemme 3.2. Pour tout $s \in I_{N}$, on a:

$$
\check{K}_{N}^{s}=\frac{\sqrt{3}}{24}\left|\frac{f^{\prime \prime}(s)}{f^{\prime}(s)}\right|^{4}\left[\left|\frac{f^{\prime}(s) f^{\prime \prime \prime}(s)}{f^{\prime \prime}(s)^{2}}\right|^{2}-\Re\left(\frac{f^{\prime}(s) f^{\prime \prime \prime}(s)}{f^{\prime \prime}(s)^{2}}\right)\right] \frac{1}{N^{4}}+O\left(\frac{1}{N^{6}}\right)
$$

quand $N \longrightarrow \infty$, uniformément sur $I_{N}$.

Démonstration du lemme 3.2. L'essentiel du travail a déjà été fait dans la section VI de $[\mathrm{CM}]$ (voir le lemme 1 et la remarque qui le suit). On y démontre l'existence d'une constante $c=c_{f}(s)$ telle que

$$
\check{K}_{N}^{s}=\frac{c}{N^{4}}+O\left(\frac{1}{N^{6}}\right)
$$

et que cette constante est de la forme

$c_{f}(s)=\lambda\left[\Re\left(f^{\prime \prime}(s)^{2} \overline{f^{\prime \prime \prime}(s)}\right)-\left|f^{\prime \prime \prime}(s)\right|^{2}\right]+\mu \Im\left(f^{\prime \prime}(s)^{2} \overline{f^{\prime \prime \prime}(s)}\right)+v\left[\left|f^{\prime \prime}(s)\right|^{4}-\left|f^{\prime \prime \prime}(s)\right|^{2}\right]$

lorsque $f$ est normalisée pour que $f^{\prime}(s)=1$, les constantes $\lambda, \mu, v$ ne dépendant ni de $f$, ni de $s$.

Pour les calculer, on commence par remarquer que $\check{K}_{N}^{s}$ ne change pas lorsqu'on remplace $f$ par $\bar{f}$, de sorte que $\mu=0$. En calculant directement $c_{f}(0)$ pour $f(z)=$ $\frac{1}{2}(1+z)^{2}$, on trouve $c_{f}(0)=v=0$, et pour $f$ telle que $f^{\prime}(z)=\exp \left(\frac{1}{2} z^{2}\right)$, on trouve $c_{f}(0)=-\lambda=\sqrt{3} / 24$. Il reste ensuite à remplacer $f$ par $f / f^{\prime}(s)$ dans l'expression de $c_{f}(s)$ pour obtenir l'expression voulue.

Ce lemme permet de réécrire le système vérifié par $\dot{u}_{N}(t)$, à l'aide de la fonction $\check{f}$ introduite dans la section 2, sous la forme:

$$
\begin{cases}\Delta_{N} \dot{u}_{N}(s)=\frac{3 \sqrt{3}}{2 N^{4}} \check{f}(s)+O\left(\frac{1}{N^{6}}\right), & \forall s \in I_{N}, \\ \dot{u}_{N}^{s}=0, & \forall s \in B_{N} .\end{cases}
$$

\section{Principe du maximum pour les empilements non plats}

L'objet de cette section est d'obtenir des estimations a priori sur les rayons $r_{N}^{s}(t)$ en vue de contrôler l'inverse du laplacien discret $\Delta_{N}$. Ces estimations font l'objet du corollaire 4.3 
dont le lecteur pressé peut tout de suite prendre connaissance avant de passer à la section suivante.

Un résultat couramment utilisé pour comparer les rayons des cercles de deux empilements de même combinatoire est le lemme de Schwarz-Pick discret (voir [BS], [CM] Section V, et [M] Section III). Malheureusement, ce type de résultat ne s'applique pas dans la situation présente, puisque nous étudions des empilements de cercles tracées sur des variétés euclidiennes non immergées isométriquement, car pourvues de singularités coniques. Que se passe-t-il lorsque la courbure en les sommets intérieurs varie? La réponse est fournie par la

Proposition 4.1. Soit $\mathcal{T}$ une triangulation finie d'un disque topologique, $S$ (resp. $B, I)$ l'ensemble des sommets de $\mathcal{T}$ (resp. sommets du bord, sommets intérieurs), soit $r=\left(r_{s}\right)_{s \in S}$ et $r^{\prime}=\left(r_{s}^{\prime}\right)_{s \in S} \in\left(\mathbf{R}_{+}^{*}\right)^{S}$, et $\mathcal{T}_{r}, \mathcal{T}_{r^{\prime}}$ les variété euclidiennes à singularités coniques définies à partir de $\mathcal{T}, r$ et $r^{\prime}$. On suppose que:

(i) $\forall s \in B, r_{s}=r_{s}^{\prime}$,

(ii) $\forall s \in I, K_{s}(r) \leq K_{s}\left(r^{\prime}\right)$.

Alors on a $\forall s \in I, r_{s} \leq r_{s}^{\prime}$, et si il y a égalité pour un sommet, il y a égalité pour tout sommet.

Preuve de la proposition 4.1. On réalise la variété $\mathcal{T}_{r^{\prime}}$ comme le temps 1 d'une déformation $\left\{\mathcal{T}_{r}(t), t \in[0,1]\right\}$ de la variété $\mathcal{T}_{r}$ exactement comme dans la section précédente. En particulier, la courbure en tout sommet intérieur $s$ de $\mathcal{T}_{r}(t)$ est définie par

$$
K_{s}(t)=(1-t) K_{s}(r)+t K_{s}\left(r^{\prime}\right) .
$$

Notons $u_{s}(t)=\log r_{s}(t)$. Pour tout $t$, il existe un laplacien discret $\Delta^{t} \operatorname{sur} \mathcal{T}$ (au sens du Section 3) tel que

$$
\begin{cases}\Delta^{t} \dot{u}(s)=K_{s}\left(r^{\prime}\right)-K_{s}(r), & \forall s \in I, \\ \dot{u}_{s}(t)=0, & \forall s \in B .\end{cases}
$$

Pour $s \in I$ et $s^{\prime} \in S$ voisin de $s$, notons $p_{s s^{\prime}}^{t}=-\left(\partial K_{s}^{t} / \partial u_{s^{\prime}}\right) /\left(\partial K_{s}^{t} / \partial u_{s}\right)$. On rappelle que $\left.p_{s s^{\prime}}^{t} \in\right] 0,1\left[\right.$ et que, si $s \in I$ est fixé, alors $\sum_{s^{\prime} \sim s} p_{s s^{\prime}}^{t}=1$.

Par hypothèse, $\Delta^{t} \dot{u}(s) \geq 0$ pour tout $s \in I$, ce qui signifie $\dot{u}_{s}(t) \geq \sum_{s^{\prime} \sim s} p_{s s^{\prime}}^{t} \dot{u}_{s^{\prime}}(t)$. On en déduit aussitot que, si la fonction $s \longrightarrow \dot{u}_{s}(t)$ définie sur $S$ atteint son minimum en un sommet intérieur, alors elle est constante et égale à sa valeur sur le bord, à savoir 0 . Si elle atteint son minimum en un sommet du bord, ce minimum est nul, de sorte que $\dot{u}_{s}(t) \geq$ 0 pour tout $t$ et tout sommet $s \in I$. On en déduit que $\log \left(r_{s}^{\prime} / r_{s}\right)=u_{s}(1)-u_{s}(0) \geq 0$ pour tout $s \in I$, ce qui prouve la première partie de l'assertion.

La seconde partie résulte du fait que, si $r_{s_{0}}^{\prime}=r_{s_{0}}$ pour un $s_{0} \in I$, alors $\dot{u}_{s_{0}}(t)=0$, donc $\dot{u}_{s}(t)=0$ pour tout $s \in I$ comme on vient de le voir, donc $r_{s}^{\prime}=r_{s}$.

Cet énoncé, également présent dans [D2], semble la généralisation la plus naturelle du lemme de Schwarz-Pick discret. Malheureusement, il ne suffit pas, à lui seul, pour obtenir un contrôle a priori des rayons $r_{N}^{s}(t)$ de la variété $\tilde{\mathcal{T}}_{N}(t)$. En effet, il ne s'applique que si $\tilde{f}$ (cf. Section 2) garde un signe constant sur $\overline{\mathcal{U}}$, puisqu'alors $\check{K}_{N}^{s}$ ne change pas 
de signe (pour $N$ assez grand) sur $S_{N}$ de sorte que $r_{N}^{s}(t)$ est une fonction monotone de $t$ sur $[0,1]$.

Cela étant, énonçons l'estimation que l'on a en vue:

Proposition 4.2. Il existe deux constantes $k_{0} \geq 0$ et $c_{0} \geq 1$ vérifiant la propriété suivante:

pour tout $N \geq N_{0}$, pour tout $r \in\left(\mathbf{R}_{+}^{*}\right)^{S_{N}}$ définissant une métrique $g_{r}$ sur $\mathcal{T}_{N}$ telle que $\forall s \in I_{N},\left|K_{N}^{s}(r)\right| \leq k_{0} / N^{2}$, on $a$ :

$$
\max _{s \in S_{N}} r_{s} \leq c_{0} \max _{s \in B_{N}} r_{s} \quad \text { et } \quad \min _{s \in S_{N}} r_{s} \geq \frac{1}{c_{o}} \min _{s \in B_{N}} r_{s} .
$$

Corollaire 4.3. Il existe trois constantes $A, B$ et $N_{1}$ telles que, pour tout $N \geq N_{1}$, pour tout $t \in[0,1]$, pour tout $s \in S_{N}$, on ait:

$$
0<\frac{A}{N} \leq r_{N}^{s}(t) \leq \frac{B}{N}
$$

Démonstration $d u$ corollaire 4.3. D'après le lemme 3.2, il existe une constante $M$ telle que, pour tout $s \in I_{N}$ et pour tout $N \geq N_{0}$, on ait $\left|K_{N}^{s}(t)\right| \leq M / N^{4}$, de sorte que, pour $N$ assez grand $\left(\geq N_{1}\right)$, on a $\left|K_{N}^{s}(t)\right| \leq k_{0} / N^{2}$, où $k_{0}$ est la constante fournie par la proposition 4.2. Notons $\tilde{A}=\min _{z \in \overline{\mathcal{U}}}\left|f^{\prime}(z)\right|$ et $\tilde{B}=\max _{z \in \overline{\mathcal{U}}}\left|f^{\prime}(z)\right|$. Compte tenu de la valeur de $r_{N}^{s}(t)$ lorsque $s \in B_{N}$ (cf. proposition 3.1), on a, avec la proposition 4.2,

$$
\frac{1}{c_{0}} \cdot \frac{\tilde{A}}{2 N} \leq r_{N}^{s}(t) \leq c_{0} \cdot \frac{\tilde{B}}{2 N}
$$

et ce pour tous $N \geq N_{1}, t \in[0,1]$ et $s \in I_{N}$, de sorte que l'on peut prendre $A=\tilde{A} / 2 c_{0}$ et $B=c_{0} \tilde{B} / 2$.

Le reste de la section est consacré à la preuve de la proposition 4.2, que l'on obtient par voie probabiliste.

Preuve de la proposition 4.2. Le point de départ est une-modeste-généralisation de deux inégalités de convexité observées à cinq ans d'intervalle par Rodin d'une part (voir [R]), et Bárány et al. d'autre part (voir [BFP]):

Lemme 4.4. Il existe une constante D possédant la propriété suivante: soit $k \in[0, \pi]$, soit $r_{0}, \ldots, r_{6}$ sept réels $>0$, et soit, pour $1 \leq i \leq 5, \alpha_{i}$ (resp. $\left.\alpha_{6}\right)$ l'angle en 0 dans le triangle euclidien de côtés $r_{0}+r_{i}, r_{i}+r_{i+1}, r_{i+1}+r_{0}$ (resp. $\left.r_{0}+r_{6}, r_{6}+r_{1}, r_{1}+r_{0}\right)$.

$$
\text { Si }-k \leq 2 \pi-\sum_{i=1}^{6} \alpha_{i} \leq k \text {, alors }
$$

$$
\text { (a) } r_{0} \leq \frac{1+D k}{6} \sum_{i=1}^{6} r_{i} \quad \text { et } \quad \text { (b) } \frac{1}{r_{0}} \leq \frac{1+D k}{6} \sum_{i=1}^{6} \frac{1}{r_{i}} \text {. }
$$




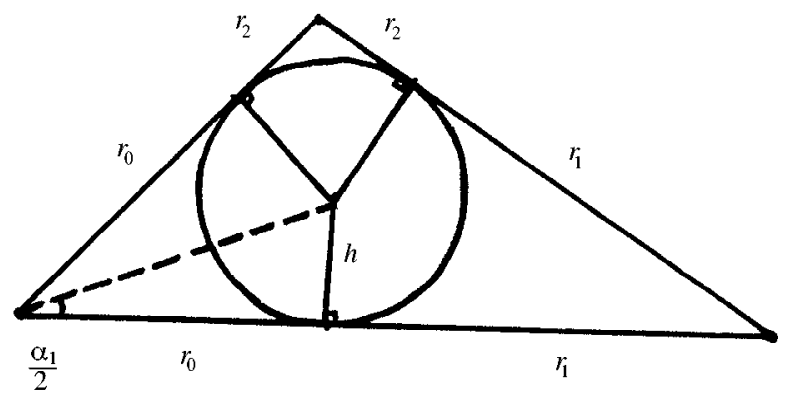

Fig. 2. Un triangle euclidien.

Démonstration du lemme 4.4. Prouvons (a). Lorsque $k=0$, ça n'est rien d'autre que le lemme 2.1 de [R]. Relisons-en la preuve pour l'adapter à la situation présente. Soit $h$ le rayon du cercle inscrit dans le triangle euclidien de côtés $r_{0}+r_{1}, r_{1}+r_{2}, r_{2}+r_{0}$ (cf. fig. 2). Il est donné par la formule

$$
h=\sqrt{\frac{r_{0} r_{1} r_{2}}{r_{0}+r_{1}+r_{2}}}
$$

de sorte que $\left.\tan \left(\alpha_{1} / 2\right)=\left(h / r_{0}\right)=\left(1 / r_{0}\right) \sqrt{r_{0} r_{1} r_{2} /\left(r_{0}+r_{1}+r_{2}\right.}\right)$.

Sachant que $\left.\alpha_{i} \in\right] 0, \pi$ [, la convexité de la fonction tangente sur ]0, $\pi / 2[$ donne:

$$
\tan \left(\frac{1}{6} \sum_{i=1}^{6} \frac{\alpha_{i}}{2}\right) \leq \frac{1}{6} \sum_{i=1}^{6} \tan \frac{\alpha_{i}}{2}
$$

Comme $2 \pi-\sum_{i=1}^{6} \alpha_{i} \leq k$, il vient:

$$
\tan \left(\frac{\pi}{6}-\frac{k}{12}\right) \leq \frac{1}{6 r_{0}}\left(\sqrt{\frac{r_{0} r_{1} r_{2}}{r_{0}+r_{1}+r_{2}}}+\cdots+\sqrt{\frac{r_{0} r_{6} r_{1}}{r_{0}+r_{6}+r_{1}}}\right) .
$$

D'après l'inégalité entre les moyennes arithmétique et géométriques, on a $\left(r_{0} r_{1} r_{2}\right)^{1 / 3} \leq$ $\frac{1}{3}\left(r_{0}+r_{1}+r_{2}\right)$, donc $r_{0} r_{1} r_{2} /\left(r_{0}+r_{1}+r_{2}\right) \leq \frac{1}{27}\left(r_{0}+r_{1}+r_{2}\right)^{2}$. Finalement, on obtient

$$
\tan \left(\frac{\pi}{6}-\frac{k}{12}\right) \leq \frac{1}{18 \sqrt{3} r_{0}}\left(6 r_{0}+2\left(r_{1}+\cdots+r_{6}\right)\right)
$$

ce qui donne

$$
r_{0} \leq \frac{c_{1}(k)}{6} \sum_{i=1}^{6} r_{i} \quad \text { avec } \quad c_{1}(k)=\frac{2}{3 \sqrt{3} \tan (\pi / 6-k / 12)-1}=1+O(k),
$$

et l'inégalité (a) est prouvée.

Prouvons (b). Lorsque $k=0$, ça n'est rien d'autre que le théorème 2 de [BFP]. Nous allons à nouveau en adapter la preuve à la situation présente. Ayant établi (cf. p. 573 de 
$[\mathrm{BFP}])$ que

$$
\frac{h}{r_{0}}\left(\tan \frac{\alpha_{1}}{4}+\operatorname{cotan} \frac{\alpha_{1}}{4}-2\right) \leq \frac{h}{r_{1}}+\frac{h}{r_{2}},
$$

il vient, en sommant,

$$
\frac{1}{6 r_{0}} \sum_{i=1}^{6}\left(\frac{1}{\sin \left(\alpha_{i} / 2\right)}-1\right)=\frac{1}{6 r_{0}} \sum_{i=1}^{6} \frac{1}{2}\left(\tan \frac{\alpha_{1}}{4}+\operatorname{cotan} \frac{\alpha_{1}}{4}-2\right) \leq \frac{1}{6} \sum_{i=1}^{6} \frac{1}{r_{i}}
$$

La convexité de $1 / \sin$ sur ]0, $\pi$ [ fournit

$$
\frac{1}{\sin \left(\frac{1}{6} \sum_{i=1}^{6}\left(\alpha_{i} / 2\right)\right)}-1 \leq \frac{1}{6} \sum_{i=1}^{6}\left(\frac{1}{\sin \left(\alpha_{i} / 2\right)}-1\right)
$$

et comme $-k \leq 2 \pi-\sum_{i=1}^{6} \alpha_{i}$, on obtient en fin de compte

$$
\frac{1}{r_{0}} \leq \frac{c_{2}(k)}{6} \sum_{i=1}^{6} \frac{1}{r_{i}} \quad \text { avec } \quad c_{2}(k)=\left[\frac{1}{\sin (\pi / 6+k / 12)}-1\right]^{-1}=1+O(k)
$$

ce qui prouve l'inégalité (b).

Le résultat suivant est un principe du maximum pour des fonctions "presque sousharmoniques" discrètes. Joint au lemme 4.4, il permettra de prouver la proposition 4.2. Rappelons que $S_{N}=B_{N} \cup I_{N}$ désigne l'ensemble des sommets de la triangulation $\mathcal{T}_{N}$.

Lemme 4.5. Il existe deux constantes $\alpha>0$ et $c_{0} \geq 1$ ayant la propriété suivante: pour toute fonction $\varphi: S_{N} \longrightarrow \mathbf{R}$ telle que

$$
\forall s \in I_{N}, \quad \varphi(s) \leq \frac{1+\alpha^{2} / N^{2}}{6} \sum_{s^{\prime} \sim s} \varphi\left(s^{\prime}\right),
$$

on $a$

$$
\max _{s \in S_{N}} \varphi(s) \leq c_{0} \cdot \max _{s \in B_{N}} \varphi(s)
$$

Admettons provisoirement ce lemme et déduisons-en la proposition 4.2. On pose $k_{0}=\min \left(\alpha^{2} / D, \pi\right)$ où $D$ est la constante fournie par le lemme 4.4. Soit $r \in\left(\mathbf{R}_{+}^{*}\right)^{S_{N}}$ tel que la métrique $g_{r}$ sur $\mathcal{T}_{N}$ vérifie $\forall s \in I_{N},\left|K_{N}^{s}(r)\right| \leq k_{0} / N^{2}$. Le lemme 4.4 permet alors d'appliquer le lemme 4.5 aux fonctions $s \longrightarrow r_{s}$ et $s \longrightarrow 1 / r_{s}$, ce qui permet de conclure.

Démonstration du lemme 4.5. Comme souvent en théorie discrète du potentiel, nous obtenons le résultat par voie probabiliste. Notons $S=(1 / N)\left(\mathbf{Z}+e^{i \pi / 3} \mathbf{Z}\right)$ l'ensemble de tous les sommets de la triangulation équilatérale $\mathcal{T}$ du plan euclidien par des triangles équilatéraux de côté $1 / N$ et contenant 0 et $1 / N$. Sur l'univers $\Omega=\{\omega=$ $\left.\left(\omega_{0}, \omega_{1}, \omega_{2}, \ldots\right) \in S^{\mathbf{N}}\right\}$ on considère la tribu cylindrique $\Sigma$ engendrée par les événements 
$\left\{\omega_{i_{1}}=s_{1}, \ldots, \omega_{i_{p}}=s_{p}\right\}$ où $p \in \mathbf{N}^{*}, i_{1}, \ldots, i_{p} \in \mathbf{N}, i_{1}<i_{2}<\cdots<i_{p}$, et $s_{1}, \ldots, s_{p} \in S$. On note $Z_{n}$ la variable aléatoire sur $(\Omega, \Sigma)$ à valeurs dans $S$ et définie $\operatorname{par} \forall \omega \in \Omega, Z_{n}(\omega)=\omega_{n}$.

Fixons un sommet $s \in S$. On définit la probabilité $P_{S} \operatorname{sur}(\Omega, \Sigma)$ associée à la marche aléatoire partant de $s$ de la façon suivante:

$$
P_{s}\left(Z_{0}=s\right)=1, \quad \text { et } \quad P_{s}\left(Z_{n+1}=v \mid Z_{n}=u\right)= \begin{cases}\frac{1}{6} & \text { si } v \sim u \\ 0 & \text { sinon. }\end{cases}
$$

Soit $\tau_{N}$ le premier temps d'atteinte du bord $B_{N}$ : c'est le temps d'arrêt défini par

$$
\tau_{N}(\omega)=\inf \left\{n \in \mathbf{N} \text { tel que } Z_{n}(\omega) \in B_{N}\right\} .
$$

On rappelle que $\tau_{N}$ est fini $P_{s}$-presque sûrement (voir par exemple le lemme 1 de la section VI de $[\mathrm{M}]$ ). Notons $\mathbf{E}_{s}$ l'espérance pour la probabilité $P_{s}$. Le lemme suivant jouera un rôle clé:

Lemme 4.6. Il existe deux constantes $\alpha>0$ et $c_{0} \geq 1$ telles que

$$
\forall N \geq 1, \quad \forall s \in S_{N}, \quad \mathbf{E}_{s}\left[\left(1+\frac{\alpha^{2}}{N^{2}}\right)^{\tau_{N}}\right] \leq c_{0} .
$$

Démonstration du lemme 4.6. On commence par se ramener à un problème unidimensionnel. Notons $Y_{n}$ l'ordonnée de $Z_{n}$ et posons $y_{n}=(2 / \sqrt{3}) Y_{n}$ : c'est une variable aléatoire $\operatorname{sur}(\Omega, \Sigma)$ à valeurs dans $(1 / N) \mathbf{Z}$. De plus, si $s=(\sqrt{3} / 2)\left(\xi_{s}+i \eta_{s}\right)$, alors on a:

$$
P_{s}\left(y_{0}=\eta_{s}\right)=1, \quad \text { et } \quad P_{s}\left(y_{n+1}=\frac{q}{N} \mid y_{n}=\frac{p}{N}\right)= \begin{cases}\frac{1}{3} & \text { si }|q-p| \leq 1 \\ 0 & \text { sinon. }\end{cases}
$$

Soit $\mathcal{B}=\{|\Im z| \leq(\sqrt{3} / 2) a\}$ une bande contenant le $N_{0}$-voisinage de l'ouvert borné $\mathcal{U}\left(\dashv>\right.$ '). Ainsi, si $\sigma_{N}=\inf \left\{n \in \mathbf{N}\right.$ tel que $\left.\left|y_{n}\right| \geq a\right\}$, alors $\tau_{N} \leq \sigma_{N} P_{s}$-presque sûrement.

Soit $\Psi$ la fonction définie sur $]-\pi / 2 a, \pi / 2 a$ [ par

$$
\Psi(t)=\frac{\mathbf{E}_{s}\left(e^{i t y_{1}}\right)}{\mathbf{E}_{s}\left(e^{i t y_{0}}\right)}=\frac{1}{3}+\frac{2}{3} \cos \frac{t}{N}
$$

et soit $\mathcal{F}_{n}$ la tribu engendrée par $y_{0}, y_{1}, \ldots, y_{n}$. On vérifie aisément que la suite $\left\{\left(\Psi(t)^{-n} e^{i t y_{n}}, \mathcal{F}_{n}\right), n \in \mathbf{N}\right\}$ est une martingale. Le théorème optionnel de Doob as-

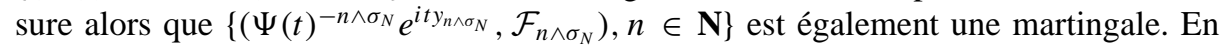
particulier, la suite des espérances est constante:

$$
\forall n \geq 0, \quad \mathbf{E}_{s}\left[\Psi(t)^{-n \wedge \sigma_{N}} e^{i t y_{n \wedge \sigma_{N}}}\right]=e^{i t \eta_{s}} .
$$

En identifiant les parties réelles des deux membres, il vient

$$
\forall n \geq 0, \quad \mathbf{E}_{s}\left[\Psi(t)^{-n \wedge \sigma_{N}} \cos \left(t y_{n \wedge \sigma_{N}}\right)\right]=\cos \left(t \eta_{s}\right) .
$$


Comme $\left|t y_{n \wedge \sigma_{N}}\right|<\pi / 2$, chaque fonction intégrée est positive, donc, grâce au lemme de Fatou, on a:

$$
\mathbf{E}_{s}\left[\Psi(t)^{-\sigma_{N}} \cos \left(t y_{\sigma_{N}}\right)\right] \leq \cos \left(t \eta_{s}\right) \leq 1,
$$

et comme $\left|y_{\sigma_{N}}\right|=a$, il vient finalement $\mathbf{E}_{s}\left[\Psi(t)^{-\sigma_{N}}\right] \leq 1 / \cos (a t)$. Compte tenu du fait que $\Psi(t)^{-1}=\left(\frac{1}{3}+\frac{2}{3} \cos (t / N)\right)^{-1} \geq 1+t^{2} / 4 N^{2}$ pour $\left.t \in[-2 \alpha, 2 \alpha] \subset\right]-$ $\pi / 2 a, \pi / 2 a$ [ et que $\tau_{N} \leq \sigma_{N} P_{s}$-presque sûrement, on en déduit l'inégalité voulue avec $c_{0}=1 / \cos 2 a \alpha$.

Fin de la preuve du lemme 4.5. Nous allons montrer que les constantes $\alpha$ et $c_{0}$ fournies par le lemme 4.6 conviennent. Soit donc $\varphi: S_{N} \longrightarrow \mathbf{R}$ telle que

$$
\forall s \in I_{N}, \quad \varphi(s) \leq \frac{1+\alpha^{2} / N^{2}}{6} \sum_{s^{\prime} \sim s} \varphi\left(s^{\prime}\right) .
$$

On a $\varphi(s) \leq\left(1+\alpha^{2} / N^{2}\right) \mathbf{E}_{s} \varphi\left(Z_{1}\right)$, puis (récurrence) $\varphi(s) \leq\left(1+\alpha^{2} / N^{2}\right)^{n} \mathbf{E}_{s} \varphi\left(Z_{n}\right)$. Plus généralement, on a l'inégalité suivante (comparer avec le lemme 2 de la section VI de $[\mathrm{M}])$ :

$$
\varphi(s) \leq \mathbf{E}_{s}\left[\left(1+\frac{\alpha^{2}}{N^{2}}\right)^{\tau_{N}} \varphi\left(Z_{\tau_{N}}\right)\right] .
$$

Prouvons cette inégalité. On commence par observer que la suite $\left\{\left(\left(1+\alpha^{2} / N^{2}\right)^{n \wedge \tau_{N}} \varphi\left(Z_{n \wedge \tau_{N}}\right), \mathcal{F}_{n}\right), n \in \mathbf{N}\right\}$ est une surmartingale, de sorte que la suite des espérances est croissante. On en déduit:

$$
\forall n \geq 0, \quad \varphi(s) \leq \mathbf{E}_{s}\left[\left(1+\frac{\alpha^{2}}{N^{2}}\right)^{n \wedge \tau_{N}} \varphi\left(Z_{n \wedge \tau_{N}}\right)\right]
$$

Soit $M=\max _{s \in S_{N}}|\varphi(s)|$. On a, pour tout $n \geq 0$,

$$
\left|\left(1+\frac{\alpha^{2}}{N^{2}}\right)^{n \wedge \tau_{N}} \varphi\left(Z_{n \wedge \tau_{N}}\right)\right| \leq M\left(1+\frac{\alpha^{2}}{N^{2}}\right)^{\tau_{N}},
$$

et le second membre est une variable aléatoire $P_{s}$-intégrable d'après le lemme 4.6. Le théorème de convergence dominée permet alors d'obtenir l'inégalité $(*)$ en faisant $n \longrightarrow \infty$ dans l'inégalité $(* *)$.

Comme $Z_{\tau_{N}} \in B_{N}$, on a alors

$$
\forall s \in S_{N}, \quad \varphi(s) \leq \mathbf{E}_{s}\left[\left(1+\frac{\alpha^{2}}{N^{2}}\right)^{\tau_{N}}\right] \max _{B_{N}} \varphi,
$$

ce qui, combiné au lemme 4.6, donne:

$$
\max _{S_{N}} \varphi \leq c_{0} \cdot \max _{B_{N}} \varphi
$$

terminant ainsi la preuve du lemme 4.5. 


\section{Fin de la preuve du théorème principal}

Rappelons, avec les notations de la section 3, que le vecteur $\dot{u}_{N}=\left(\dot{u}_{N}^{s}\right)_{s \in S_{N}} \in \mathbf{R}^{S_{N}}$ est solution du système suivant:

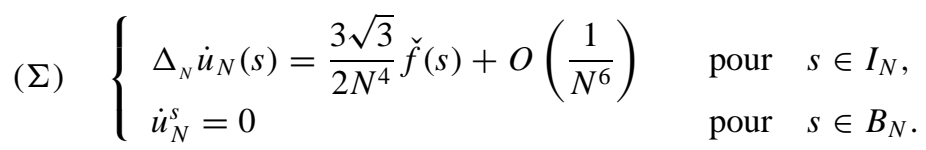

La première étape dans l'étude de la solution de ce système consiste en des

\subsection{Estimations sur l'opérateur $\Delta_{N}$}

L'opérateur $\Delta_{N}$ est un laplacien discret sur $I_{N}$, c'est-à-dire un endomorphisme symétrique défini positif de $\mathbf{R}^{I_{N}}$. Munissons $\mathbf{R}^{I_{N}}$ de la norme $L^{2}$ donnée par $\|u\|_{2}=$ $\sqrt{\sum_{s \in I_{N}} u(s)^{2}}$, et $\operatorname{End}\left(\mathbf{R}^{I_{N}}\right)$ de la norme d'opérateur associée. Si $0<\lambda_{1}^{(N)} \leq \lambda_{2}^{(N)} \leq$ $\cdots \leq \lambda_{p}^{(N)}$ sont les valeurs propres de $\Delta_{N}$, alors $\left\|\Delta_{N}\right\|_{2}=\lambda_{p}^{(N)}$ et $\left\|\Delta_{N}^{-1}\right\|_{2}=1 / \lambda_{1}^{(N)}$.

Lemme 5.1.1. Il existe une constante $C$ ne dépendant que de $f$ telle que, pour tout $N \geq N_{1}$, on ait

$$
\left\|\Delta_{N}^{-1}\right\|_{2} \leq C \cdot N^{2}
$$

Démonstration du lemme 5.1.1. On cherche une estimation du type $\lambda_{1}^{(N)} \geq 1 / C N^{2}$, ce qui est exactement le lemme 2 de la section VI de [CM]. Vérifions qu'on peut l'appliquer ici. La seule chose à voir est l'existence d'une constante $c_{1}$ telle que, si $s \in I_{N}$ et si $s^{\prime} \sim s$, alors $-\partial K_{N}^{s} / \partial u_{N}^{s^{\prime}} \geq c_{1}>0$. Mais ceci provient de l'existence d'une constante $c_{2}$ telle que

$$
\forall N \geq N_{1}, \quad \forall s, s^{\prime} \in S_{N}, \quad \forall t \in[0,1], \quad \frac{1}{c_{2}} \leq \frac{r_{N}^{s}(t)}{r_{N}^{s^{\prime}}(t)} \leq c_{2},
$$

existence que l'on déduit immédiatement du corollaire 4.3. Le reste de la preuve est identique à celle du lemme 2 de la section VI de [CM].

Ce lemme permet d'améliorer l'estimation des rayons $r_{N}^{s}(t)$, et donc des coefficients de $\Delta_{N}$ :

Corollaire 5.1.2. On a, pour tout $s \in S_{N}$ et tout $t \in[0,1]$, les estimations uniformes suivantes:

(i) $r_{N}^{s}(t)=\left|f^{\prime}(s)\right| / 2 N+O\left(1 / N^{2}\right)$;

(ii) si $s \in I_{N}$ et si $s \sim s^{\prime}$, alors $\partial K_{N}^{s} / \partial u_{N}^{s^{\prime}}=-\sqrt{3} / 3+O(1 / N)$.

Démonstration du corollaire 5.1.2. Prouvons (i). D'après le système $(\Sigma)$ vérifié par $\dot{u}_{N}$, on a, pour tout $s \in I_{N}, \Delta_{N} \dot{u}_{N}(s)=O\left(1 / N^{4}\right)$, et comme Card $I_{N}=O\left(N^{2}\right)$, 
il vient:

$$
\left\|\Delta_{N} \dot{u}_{N}\right\|_{2}=\sqrt{\sum_{s \in I_{N}}\left(\Delta_{N} \dot{u}_{N}\right)(s)^{2}}=\sqrt{O\left(N^{2} \times \frac{1}{N^{8}}\right)}=O\left(\frac{1}{N^{3}}\right),
$$

donc, avec le lemme 5.1.1, $\left\|\dot{u}_{N}(t)\right\|_{2} \leq\left\|\Delta_{N}^{-1}\right\|_{2} \cdot\left\|\Delta_{N} \dot{u}_{N}\right\|_{2}=O\left(N^{2} \times 1 / N^{3}\right)=$ $O(1 / N)$. Il existe donc une constante $M$ telle que

$$
\forall N \geq N_{1}, \quad \forall t \in[0,1], \quad \sqrt{\sum_{s \in I_{N}} \dot{u}_{N}^{s}(t)^{2}} \leq \frac{M}{N} .
$$

On en déduit que, pour tout $s \in I_{N},\left|\dot{u}_{N}^{s}(t)\right| \leq M / N$, de sorte qu'en intégrant entre 0 et $t$,

$$
\left|u_{N}^{s}(t)-u_{N}^{s}(0)\right|=\left|\log \frac{2 N r_{N}^{s}(t)}{\left|f^{\prime}(s)\right|}\right| \leq \frac{M}{N},
$$

d'où l'estimation annoncée.

Prouvons (ii). Si $s \sim s^{\prime}$, alors on a, en notant $s_{1}$ et $s_{2}$ les sommets de $\mathcal{T}_{N}$ voisins chacun de $s$ et $s^{\prime}$,

$$
\frac{\partial K_{N}^{s}}{\partial u_{N}^{s^{\prime}}}=-\frac{1}{r_{N}^{s}+r_{N}^{s^{\prime}}}\left(\sqrt{\frac{r_{N}^{s} r_{N}^{s^{\prime}} r_{N}^{s^{1}}}{r_{N}^{s}+r_{N}^{s^{\prime}}+r_{N}^{s^{1}}}}+\sqrt{\frac{r_{N}^{s} r_{N}^{s^{\prime}} r_{N}^{s^{2}}}{r_{N}^{s}+r_{N}^{s^{\prime}}+r_{N}^{s^{2}}}}\right) .
$$

Comme $\left|s-s^{\prime}\right|=1 / N$, on a, avec le point (i),

$$
\frac{\partial K_{N}^{s}}{\partial u_{N}^{s^{\prime}}}=-\frac{\sqrt{3}}{3}+O\left(\frac{1}{N}\right) .
$$

\subsection{Emploi de la méthode des volumes finis}

Lorsqu'on écrit le système $(\Sigma)$ sous la forme

$$
\begin{cases}\Delta_{N}\left(N^{2} \dot{u}_{N}\right)(s)=\frac{3 \sqrt{3}}{2 N^{2}} \check{f}(s)+O\left(\frac{1}{N^{4}}\right) & \text { pour } \quad s \in I_{N}, \\ N^{2} \dot{u}_{N}^{s}=0 & \text { pour } s \in B_{N},\end{cases}
$$

il s'apparente à une discrétisation du problème de Dirichlet

$$
\text { (П) } \begin{cases}\Delta g=\check{f} & \operatorname{sur} \mathcal{U}, \\ g=0 & \text { sur } \partial \mathcal{U} .\end{cases}
$$

L'objet de ce paragraphe est d'achever la preuve du théorème principal en estimant la convergence de $N^{2} \dot{u}_{N}$ vers $g$. Voici l'estimation que l'on a en vue:

Proposition 5.2.1. On a, pour tout $s \in S_{N}$ et tout $t \in[0,1]$, l'estimation uniforme suivante:

$$
N^{2} \dot{u}_{N}^{s}(t)=g(s)+O\left(\frac{\log N}{N}\right) .
$$


On en déduit aussitôt, en intégrant entre 0 et 1 , que

$$
u_{N}^{s}(1)-u_{N}^{s}(0)=\log \frac{2 N r_{N}^{s}}{\left|f^{\prime}(s)\right|}=\frac{g(s)}{N^{2}}+O\left(\frac{\log N}{N^{3}}\right)
$$

d'où:

$$
r_{N}^{s}=\frac{\left|f^{\prime}(s)\right|}{2 N}\left(1+\frac{g(s)}{N^{2}}\right)+O\left(\frac{\log N}{N^{4}}\right) \quad \text { quand } \quad N \longrightarrow \infty,
$$

uniformément sur $S_{N}$, ce qui est bien le développement asymptotique énoncé dans le théorème 2.1. Nous terminons ce paragraphe par la

Démonstration de la proposition 5.2.1. Celle-ci repose sur l'utilisation de la méthode dite des volumes finis. Nous allons montrer comment elle permet de rendre compte efficacement de la discrétisation $(\Sigma)$ du problème $(\Pi)$.

Considérons la cellulation duale de la triangulation $\mathcal{T}_{N}$. C'est une collection $\Xi_{N}=$ $\left\{\sigma_{s}, s \in S_{N}\right\}$ d'hexagones réguliers de côté $h=\sqrt{3} / 3 N$ et ayant chacun deux arêtes parallèles à l'axe imaginaire pur (cf. fig. 3). On note $\Xi_{N}^{I}=\left\{\sigma_{s}, s \in I_{N}\right\}$.

Le point de départ de la méthode des volumes finis consiste à intégrer l'équation $\Delta g=\breve{f}$ sur chaque hexagone de la cellulation $\Xi_{N}^{I}$ de manière à obtenir

$$
\forall s \in I_{N}, \quad-\int_{\partial \sigma_{s}} \nabla g \cdot n=\int_{\sigma_{s}} \check{f}
$$

où $\nabla g$ désigne le gradient de $g$ et $n$ la normale extérieure au bord $\partial \sigma_{s}$ de $\sigma_{s}$.

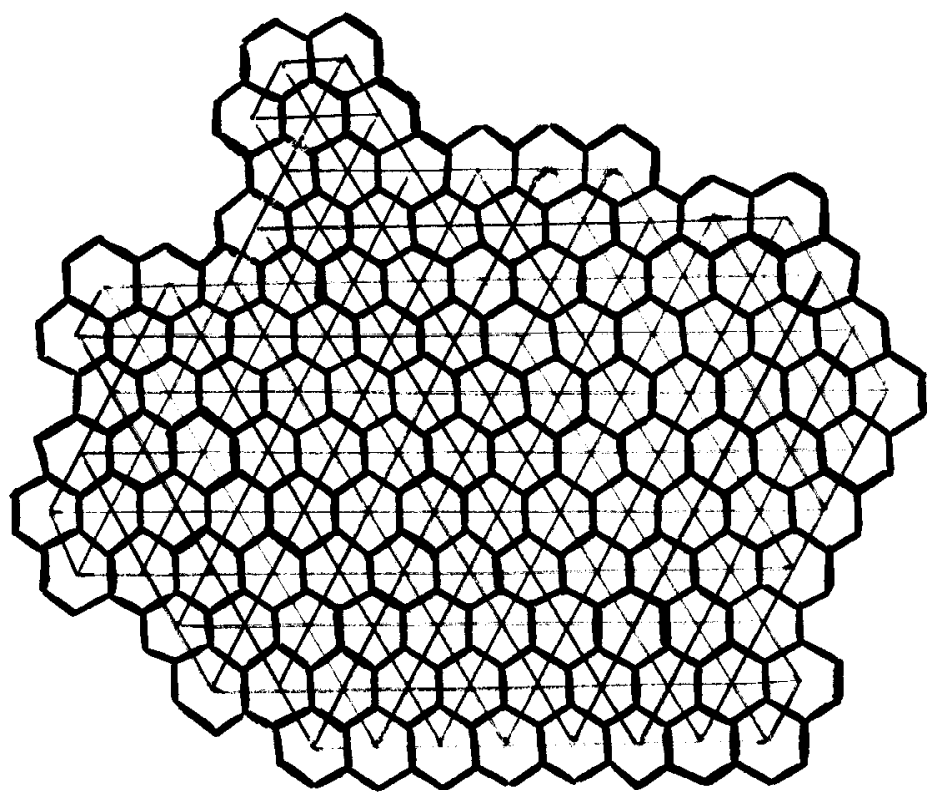

Fig. 3. La triangulation $\mathcal{T}_{N}$ avec, en gras, la cellulation $\Xi_{N}$. 


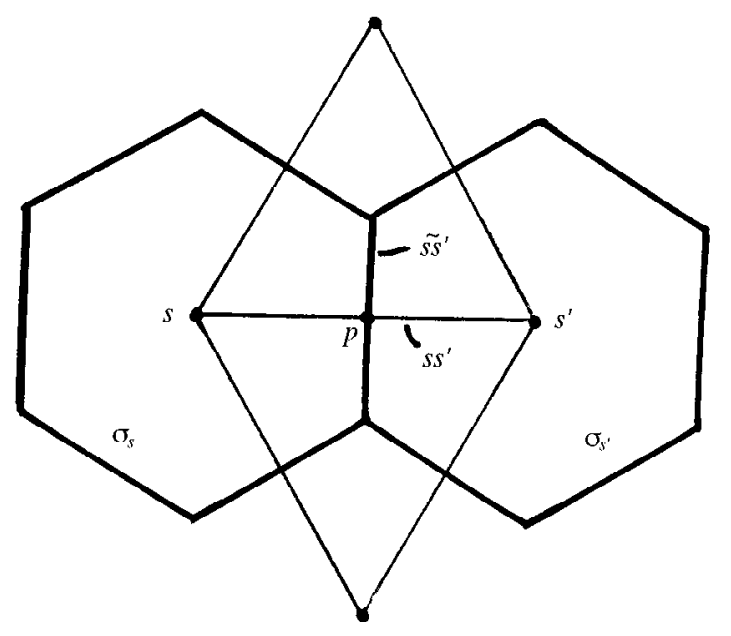

Fig. 4. Deux cellules adjacentes $\sigma_{s}$ et $\sigma_{s^{\prime}}$ et les arêtes $s s^{\prime}$ et $\tilde{s s^{\prime}}$.

Notons $\tilde{s s^{\prime}}$ l'arête commune aux deux cellules $\sigma_{s}$ et $\sigma_{s^{\prime}}$ (cf. fig. 4), et introduisons les deux flux

$$
\begin{gathered}
\left.\bar{F}_{s \rightarrow s^{\prime}}=-\int_{\tilde{s}^{\prime}} \nabla g \cdot n \text { (la normale } n \text { est orientée de } s \text { vers } s^{\prime}\right), \\
\text { et } \quad F_{s \rightarrow s^{\prime}}^{*}=c_{s s^{\prime}}\left(g\left(s^{\prime}\right)-g(s)\right) \quad \text { où } \quad c_{s s^{\prime}}=\frac{\partial K_{N}^{s}}{\partial u_{N}^{s^{\prime}}} .
\end{gathered}
$$

La quantité $F_{s \rightarrow s^{\prime}}^{*}$ se révèle être une approximation du flux exact $\bar{F}_{s \rightarrow s^{\prime}}$, comme en témoigne le

Lemme 5.2.2. Pour $s \in I_{N}$ et $s^{\prime} \in S_{N}$ voisin de $s$, on a l'estimation uniforme suivante:

$$
R_{s \rightarrow s^{\prime}} \stackrel{\text { def }}{=} \bar{F}_{s \rightarrow s^{\prime}}-F_{s \rightarrow s^{\prime}}^{*}=O\left(\frac{1}{N^{2}}\right) .
$$

Démonstration du lemme 5.2.2. Tout d'abord, on a, d'après le corollaire 5.1.2, $c_{s s^{\prime}}=$ $\partial K_{N}^{s} / \partial u_{N}^{s^{\prime}}=-1 / \sqrt{3}+O(1 / N)$. Puisque $\left|s-s^{\prime}\right|=1 / N$, et que $g$ est de classe $C^{1}$ (en fait $\left.C^{2}\right) \operatorname{sur} \overline{\mathcal{U}}$, on a

$$
F_{s \rightarrow s^{\prime}}^{*}=-\frac{1}{\sqrt{3}}\left(g\left(s^{\prime}\right)-g(s)\right)+O\left(\frac{1}{N^{2}}\right) .
$$

Notons $p$ le point d'intersection de l'arête $s s^{\prime}$ et de l'arête $\tilde{s}^{\prime}$ (cf. fig. 4). Un développement de Taylor à l'ordre 2 fournit d'une part

$$
g\left(s^{\prime}\right)-g(s)=\nabla g(p) \cdot n \times \operatorname{long}\left(s s^{\prime}\right)+O\left(\frac{1}{N^{2}}\right),
$$


et d'autre part

$$
\int_{\tilde{s s^{\prime}}} \nabla g \cdot n=\nabla g(p) \cdot n \times \operatorname{long}\left(\tilde{s} \tilde{s}^{\prime}\right)+O\left(\frac{1}{N^{2}}\right) .
$$

Comme $\operatorname{long}\left(s s^{\prime}\right)=\sqrt{3} \operatorname{long}\left(\tilde{s s^{\prime}}\right)$, on a

$$
\frac{1}{\sqrt{3}}\left(g\left(s^{\prime}\right)-g(s)\right)-\int_{\tilde{s s^{\prime}}} \nabla g \cdot n=O\left(\frac{1}{N^{2}}\right),
$$

d'où le résultat.

Réécrivons donc le problème (П) sous la forme

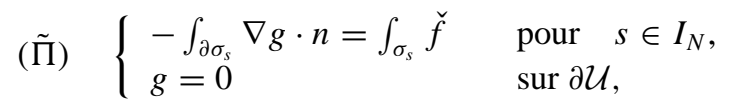

et discrétisons-le de cette manière, c'est-à-dire notons $v_{N}$ la solution de:

$$
(\tilde{\Sigma}) \quad \begin{cases}\Delta_{N} v_{N}(s)=\sum_{s^{\prime} \sim s} c_{s s^{\prime}}\left(v_{N}^{s^{\prime}}-v_{N}^{s}\right)=\int_{\sigma_{s}} \check{f} & \text { pour } \quad s \in I_{N}, \\ v_{N}^{s}=0 & \text { pour } \quad s \in B_{N} .\end{cases}
$$

Il reste alors à estimer l'écart entre $v_{N}$ et $g$ d'une part, et l'écart entre $v_{N}$ et $N^{2} \dot{u}_{N}$ d'autre part.

Lemme 5.2.3. On a, pour $s \in S_{N}$, l'estimation uniforme suivante:

$$
v_{N}^{s}-N^{2} \dot{u}_{N}^{s}=O\left(\frac{1}{N}\right)
$$

Démonstration du lemme 5.2.3. Nous allons évaluer l'image du vecteur $v_{N}-N^{2} \dot{u}_{N}$ par l'opérateur $\Delta_{N}$. Pour $s \in I_{N}$, on a:

$$
\Delta_{N}\left(v_{N}-N^{2} \dot{u}_{N}\right)(s)=\int_{\sigma_{s}} \check{f}-\frac{3 \sqrt{3}}{2 N^{2}} \check{f}(s)+O\left(\frac{1}{N^{4}}\right) .
$$

Comme $s$ est le centre de gravité de l'hexagone $\sigma_{s}$, on a, avec un développement de Taylor à l'odre 2,

$$
\int_{\sigma_{s}} \check{f}=\operatorname{Aire}\left(\sigma_{s}\right)\left[\check{f}(s)+O\left(\frac{1}{N^{2}}\right)\right]=\frac{3 \sqrt{3}}{2 N^{2}} \check{f}(s)+O\left(\frac{1}{N^{4}}\right),
$$

d'où $\Delta_{N}\left(v_{N}-N^{2} \dot{u}_{N}\right)(s)=O\left(1 / N^{4}\right)$. Exactement comme dans la preuve du corollaire 5.1.2, en utilisant l'estimation $\left\|\Delta_{N}^{-1}\right\|_{2}=O\left(N^{2}\right)$ du lemme 5.1.1, on en déduit que $v_{N}^{s}-N^{2} \dot{u}_{N}^{s}=O(1 / N)$ pour tout $s \in S_{N}$. 
Les estimations d'erreurs de la méthode des volumes finis vont permettre de mesurer l'écart entre $v_{N}$ et $g$. Notons $e$ la fonction "erreur" définie sur $S_{N} \operatorname{par} e(s)=v_{N}^{s}-g(s)$. La non-nullité éventuelle de $e$ sur $B_{N}$ se révèlera gênante pour obtenir une estimation de Sobolev $H_{0}^{1}$ discrète, ce qui est le point de départ des calculs d'erreurs dans la méthode des volumes finis.

Pour contourner cet écueil, on introduit la fonction $e^{*}$ définie par

$$
\begin{cases}\Delta_{N} e^{*}(s)=0 & \text { pour } \quad s \in I_{N}, \\ e^{*}(s)=e(s) & \text { pour } \quad s \in B_{N},\end{cases}
$$

et enfin la fonction $\tilde{e}=e-e^{*}$. Cette perturbation de $e$ est inoffensive. En effet, comme chaque sommet de $B_{N}$ est à distance $\leq 1 / N$ de $\partial \mathcal{U}$, et que $g$ est nulle sur $\partial \mathcal{U}$, il existe une constante $M$ telle que, pour tout $s \in B_{N}$, on ait $|e(s)|=\left|e^{*}(s)\right| \leq M / N$. D'après le principe du maximum pour les fonctions harmoniques discrètes, on a

$$
\left|e^{*}(s)\right|=|\tilde{e}(s)-e(s)| \leq \frac{M}{N} \quad \text { pour tout } \quad s \in S_{N} .
$$

Lemme 5.2.4 (Estimation de Sobolev discrète). Il existe une constante $C$ ne dépendant que de $f$ telle que, pour tout $N \geq N_{1}$,

$$
\sum_{s, s^{\prime} \in S_{N}, s \sim s^{\prime}}\left(\tilde{e}(s)-\tilde{e}\left(s^{\prime}\right)\right)^{2} \leq \frac{C}{N^{2}} .
$$

Démonstration du lemme 5.2.4. Il s'agit d'évaluer l'énergie de $\tilde{e}$, c'est-à-dire la quantité $\left\langle\Delta_{N} \tilde{e} \mid \tilde{e}\right\rangle$.

Tout d'abord, puisque $\tilde{e}$ est nulle sur $B_{N}$, on a

$$
\left\langle\Delta_{N} \tilde{e} \mid \tilde{e}\right\rangle=\sum_{s \in I_{N}} \Delta_{N} \tilde{e}(s) \cdot \tilde{e}(s)=-\frac{1}{2} \sum_{s \sim s^{\prime}} c_{s s^{\prime}}\left(\tilde{e}(s)-\tilde{e}\left(s^{\prime}\right)\right)^{2},
$$

$\operatorname{donc}\left\langle\Delta_{N} \tilde{e} \mid \tilde{e}\right\rangle \geq\left(c_{1} / 2\right) \sum_{s^{\prime} s^{\prime}}\left(\tilde{e}(s)-\tilde{e}\left(s^{\prime}\right)\right)^{2}$, où $c_{1}$ est la constante fournie par la preuve du lemme 5.1.1.

Par ailleurs, on a:

$$
\Delta_{N} \tilde{e}(s)=\Delta_{N} e(s)=\Delta_{N} v_{N}(s)-\Delta_{N} g(s)=\int_{\sigma_{s}} \check{f}-\Delta_{N} g(s)=\sum_{s^{\prime} \sim s} R_{s \rightarrow s^{\prime}}
$$

donc

$$
\begin{aligned}
\left\langle\Delta_{N} \tilde{e} \mid \tilde{e}\right\rangle=\sum_{s \in I_{N}} \sum_{s \sim s^{\prime}} R_{s \rightarrow s^{\prime}} \cdot \tilde{e}(s) & =\frac{1}{2} \sum_{s \sim s^{\prime}}\left(\tilde{e}(s) R_{s \rightarrow s^{\prime}}+\tilde{e}\left(s^{\prime}\right) R_{s^{\prime} \rightarrow s}\right) \\
& \leq \frac{1}{2} \sum_{s \sim s^{\prime}}\left|R_{s \rightarrow s^{\prime}}\right| \cdot\left|\tilde{e}(s)-\tilde{e}\left(s^{\prime}\right)\right|
\end{aligned}
$$

car $R_{s \rightarrow s^{\prime}}=-R_{s^{\prime} \rightarrow s}$. Grâce à l'inégalité de Cauchy-Schwarz, on a:

$$
\left\langle\Delta_{N} \tilde{e} \mid \tilde{e}\right\rangle \leq \frac{1}{2} \sqrt{\sum_{s \sim s^{\prime}} R_{s \rightarrow s^{\prime}}^{2}} \cdot \sqrt{\sum_{s \sim s^{\prime}}\left(\tilde{e}(s)-\tilde{e}\left(s^{\prime}\right)\right)^{2}} .
$$


En conclusion, il vient:

$$
\frac{c_{1}}{2} \sum_{s \sim s^{\prime}}\left(\tilde{e}(s)-\tilde{e}\left(s^{\prime}\right)\right)^{2} \leq \frac{1}{2} \sqrt{\sum_{s \sim s^{\prime}} R_{s \rightarrow s^{\prime}}^{2}} \cdot \sqrt{\sum_{s \sim s^{\prime}}\left(\tilde{e}(s)-\tilde{e}\left(s^{\prime}\right)\right)^{2}},
$$

donc

$$
\sum_{s \sim s^{\prime}}\left(\tilde{e}(s)-\tilde{e}\left(s^{\prime}\right)\right)^{2} \leq \frac{1}{c_{1}^{2}} \sum_{s \sim s^{\prime}} R_{s \rightarrow s^{\prime}}^{2} .
$$

Le membre de droite de cette inégalité est une somme de $O\left(N^{2}\right)$ termes, chacun d'eux étant un $O\left(1 / N^{4}\right)$ d'après le lemme 5.2.2, ce qui donne la majoration souhaitée.

Cette estimation de Sobolev discrète permet d'accéder à une estimation $L^{q}$ discrète suivant un procédé standard en méthode des volumes finis (voir le lemme 3.4 et le corollaire $3.1 \mathrm{de}[\mathrm{EGH}]$ ainsi que le corollaire $1 \mathrm{de}[\mathrm{CGH}])$ :

Corollaire 5.2.5 (Estimation $L^{q}$ discrète, $q \geq 1$ ). Il existe une constante $C^{\prime}$ ne dépendant que de $f$ telle que, pour tout $N \geq N_{1}$ et tout $q \in[1,+\infty[$, on ait

$$
\left(\sum_{s \in S_{N}} \operatorname{Aire}\left(\sigma_{s}\right)|\tilde{e}(s)|^{q}\right)^{1 / q} \leq \frac{C^{\prime} \cdot q}{N} .
$$

Puisque Aire $\left(\sigma_{s}\right)=3 \sqrt{3} / 2 N^{2}$ (et que $3 \sqrt{3} / 2>1$ ), on en déduit que

$$
\max _{s \in S_{N}}|\tilde{e}(s)| \leq\left(\sum_{s \in S_{N}}|\tilde{e}(s)|^{q}\right)^{1 / q} \leq C^{\prime} \cdot q \cdot N^{2 / q-1} .
$$

Si $N$ est fixé, le minimum de la fonction $q \longrightarrow q N^{2 / q-1}$ sur $[1,+\infty$ [ est atteint pour $q=2 \log N$, et vaut $2 e \cdot(\log N) / N$, de sorte que

$$
\max _{s \in S_{N}}|\tilde{e}(s)|=O\left(\frac{\log N}{N}\right) .
$$

Finalement, on a:

$$
\begin{aligned}
N^{2} \dot{u}_{N}^{s}(t)-g(s) & =\left(N^{2} \dot{u}_{N}^{s}(t)-v_{N}^{s}\right)+e^{*}(s)+\tilde{e}(s) \\
& =O\left(\frac{1}{N}\right)+O\left(\frac{1}{N}\right)+O\left(\frac{\log N}{N}\right)=O\left(\frac{\log N}{N}\right),
\end{aligned}
$$

uniformément en $s \in S_{N}$ et $t \in[0,1]$, ce qui achève la preuve de la proposition 5.2.1.

\section{Remerciements}

Je remercie Richard Kenyon, Didier Piau et Marie-Hélène Vignal pour les fructueuses conversations dont ce travail a bénéficié, Philip Bowers et Ken Stephenson pour m'avoir invité à l'exposer à Cambridge, ainsi que Sylvain Gallot pour la relecture attentive qu'il en a faite. 


\section{References}

[BFP] Bárány, I., Füredi, Z., et Pach, J., Discrete convex functions and proof of the six circle conjecture of Fejes Tóth, Canad. J. Math. 36 (1984), 569-576.

[BS] Beardon, A.-F., et Stephenson, K., The Schwarz-Pick lemma for circle packings, Illinois J. Math. 39(4) (1991), 1383-1425.

[CR] Carter, I., et Rodin, B., An inverse problem for circle packings and conformal mappings, Trans. Amer. Math. Soc. 334(2) (1992), 861-875.

[C] Colin de Verdière, Y., Un principe variationnel pour les empilements de cercles, Invent. Math. 104 (1991), 655-669.

[CM] Colin de Verdière, Y., et Mathéus, F., Empilements de cercles et approximations conformes, Actes de la Table Ronde de Géométrie Riemannienne en l'honneur de Marcel Berger, Arthur L. Besse (éd.), Collection SMF Séminaires et Congrès (no. 1) (1996), pp. 253-272.

[CGH] Coudière, Y., Gallouet, T., et Herbin, R., Discrete Sobolev and $L^{p}$ Error Estimates for Approximate Finite Volume Solutions of Convection Diffusion Equations, preprint LATP (Marseille) no. 98-13 (1998).

[D1] Dubejko, T. Approximations of analytic functions with prescribed boundary conditions by circle packing maps, Discrete Comput. Geom. 17 (1997), 67-77.

[D2] Dubejko, T. Recurrent random walks, Liouville's theorem and circle packings, Math. Proc. Cambridge Philos. Soc. 121 (1997), 531-546.

[DS] Dubejko, T., et Stephenson, K., Circle packing: experiments in discrete analytic function theory, Experimental Math. 4(4) (1995), 307-347.

[EGH] Eymard, R., Gallouët, T., et Herbin, R., The Finite Volume Method, en préparation pour The Handbook of Numerical Analysis, Ph. Ciarlet et J.L. Lions (eds.).

[GT] Gilbarg, D., et Trudinger, N.S., Elliptic Partial Differential Equations of Second Order, 2nd edn., Springer-Verlag, New York, 1983.

[HR] He, Z.X., et Rodin, B., Convergence of circle packings of finite valence to Riemann mapping, Comm. Anal. Geom. 1(1) (1993), 31-41.

[HS] He, Z.X., et Schramm, O., On the convergence of circle packings to the Riemann map, Invent. Math. 125 (1996), 285-305.

[M] Mathéus, F., Empilements de cercles et représentations conformes: une nouvelle preuve du théorème de Rodin-Sullivan, Enseign. Math. 42(1-2) (1996), 125-152.

[R] Rodin, B., Schwarz's lemma for circle packings, Invent. Math. 89 (1987), 271-289.

[RS] Rodin, B., et Sullivan, D., The convergence of circle packings to the Riemann mapping, J. Differential Geom. 26 (1987), 349-360.

[S1] Stephenson, K., Circle packings in the approximation of conformal mappings, Bull. Amer. Math. Soc. 23(2) (1990), 407-415 (Research Announcements).

[S2] Stephenson, K., A probabilistic proof of Thurston's conjecture on circle packings, Preprint, University of Tennessee, Knoxville, 1993.

[Th] Thurston, W., The finite Riemann mapping theorem, Invited talk, an International Symposium at Purdue University on the occasion of the proof of the Bieberbach Conjecture, March 1985.

[Tr] Troyanov, M., Les surfaces euclidiennes à singularités coniques, Enseign. Math. 32 (1986), 79-94.

Received August 4, 1997, and in revised form September 30, 1998. 